\title{
Properties of Inconel 625 Mesh Structures Grown by Electron Beam Additive Manufacturing
}

\author{
F. A. List ${ }^{a}{ }^{\dagger}$, R. R. Dehoff ${ }^{a}$, L. E. Lowe ${ }^{a}$, and W. J. Sames ${ }^{b}$ \\ a Oak Ridge National Laboratory, 1 Bethel Valley Rd., Oak Ridge, TN, USA \\ b Texas A\&M University, College Station, TX, USA \\ ${ }^{\dagger}$ Corresponding Author: Tel.: 1865576 8020; E-mail: listfaiii@ornl.gov
}

\begin{abstract}
Relationships between electron beam parameters (beam current, beam speed, and beam focus) and physical properties (mass, diameter, elastic modulus, and yield strength) have been investigated for Inconel 625 mesh cubes fabricated using an additive manufacturing technology based on electron beam melting. The elastic modulus and yield strength of the mesh cubes have been systematically varied by approximately a factor of ten by changing the electron beam parameters. Simple models have been used to understand these relationships. Structural anisotropies of the mesh associated with the layered build architecture have been observed and may contribute, along with microstructural anisotropies, to the anisotropic mechanical properties of the mesh. Knowledge of this kind is likely applicable to other metal and alloy systems and is essential to rapidly realize the full potential of this burgeoning technology.
\end{abstract}

\section{Keywords}

Nickel based superalloys; Inconel 625; electron beam melting; additive manufacturing; mesh structures; mechanical characterization

\section{Introduction}

Mesh structures may be incorporated into mechanical designs to tailor a variety of physical properties including mass, surface area, modulus, strength, and both thermal and electrical transport properties. This can lead to improved performance and reduced cost. For certain applications, control of the spatial gradient of a physical property is desirable. An example of such an application is a porous biomedical bone implant [1-3], where the implant must both promote osseointegration and match the varying mechanical requirements of the surrounding bone tissue. For these applications, precise, finescale control of mesh properties is generally advantageous.

Additive manufacturing (AM) based on electron beam melting (EBM) of metal powder is a particularly versatile technique to fabricate metal mesh structures with both uniform and spatially graded properties. By dividing the three dimensional mesh into two dimensional layers and sequentially building each layer, the desired three dimensional structure can be approximated. For EBM, the scale 
over which spatial control of properties is attainable is limited in part by a) the lateral dimensions of the electron beam (i.e., beam size) and b) the size of the starting metal powder particles. One challenge using EBM to build fine mesh structures near the limit where mesh size approaches either the beam size or the powder particle size is to understand the relationships between the build parameters used for each layer and the properties of the final mesh structure. Another challenge is to understand the consequences and limitations of the two dimensional layer-by-layer approximation for the three dimensional structure. This paper focusses on identifying and characterizing relationships between electron beam parameters (i.e., beam current, speed, and focus) and mesh properties for EBM of Inconel 625 for the fabrication of fine mesh structures. Simple physical models are presented to help rationalize and refine some of these relationships.

\section{Experimental}

A commercial Arcam A2 EBM machine running version 3.2 SP2 control software was used to fabricate the mesh structures. A general description of the machine and its typical operation can be found elsewhere [4]. Rotary atomized powder (-120/+325 sieve size) with a composition of commercial Inconel alloy 625 , was used for fabrication. The nominal spherical particle diameter for this commercial powder ranges from 44 to $120 \mu \mathrm{m}$.

Mesh cubes were built, sixteen at one time, on a $150 \times 150 \times 10 \mathrm{~mm}, 304 \mathrm{~L}$ stainless steel start plate. Each cube had outside dimensions of approximately $30 \times 30 \times 30 \mathrm{~mm}$ and is based on a tetrahedral, diamond structure, unit cell that is oriented such that a) all struts of the mesh make an angle of $30^{\circ}$ with planes parallel to the cube faces and $b$ ) the projections of all struts onto the faces of the cube are parallel to the face diagonal (see Fig. 1). The diameters of all struts in the build model were approximately $0.025 \mathrm{~mm}$. The opposing faces of a cube are separated by eight times the unit cell dimension. Cubes were loaded into the build file as separate models so that build parameters could be varied individually for each of the sixteen cubes. A melt theme was used for all models with power analyze off, melt hatch disabled, a $0.050 \mathrm{~mm}$ layer thickness, and a single outer contour. This theme resulted in a) a single melting event for each strut within each layer and b) strut dimensions determined by the electron beam parameters. The beam current, beam speed, and focus offset current for the outer contour were varied to determine the impact of these parameters on the mesh properties. For all cubes, the unit cell, the unit cell dimension, and the outside dimensions of the cube were constant. Specimens were prepared for microstructural examination by polishing to a $1 \mu \mathrm{m}$ diamond finish followed by etching in a solution consisting of $45 \mathrm{~mL} \mathrm{HCl}, 2.2 \mathrm{~mL} \mathrm{HNO}_{3}, 2.5 \mathrm{~mL} \mathrm{H}_{2} \mathrm{SO}_{4}$ and $0.5 \mathrm{~mL} \mathrm{HF}$.

\section{Results and Discussion}

Figure 2 shows a photograph of the first start plate containing sixteen mesh cubes. For this build, the focus offset current was $0.0 \mathrm{~mA}$ (i.e., the electron beam was fully focused) and both the beam current $(I)$ and beam speed $(V)$ were varied. After removing the residual metal powder from the cubes, the mass was measured for each cube. Figure 3 shows the results of these measurements. The relative density $\left(\boldsymbol{\rho}^{*} / \boldsymbol{\rho}_{0}\right)$ is the ratio of the mesh cube mass to the calculated mass of a $30 \times 30 \times 30 \mathrm{~mm}$ fully dense 
Inconel 625 cube $\left(\sim 228 \mathrm{~g}\right.$, where $\boldsymbol{\rho}_{0}$ is $\left.\sim 8.19 \mathrm{~g} / \mathrm{cm}^{3}\right)$. For a fixed beam current the mass decreases with increasing beam speed, and for a fixed beam speed the mass increases with increasing beam current.

When the mass is plotted versus the ratio of the beam current to the beam speed $(I / N)$, a relationship is observed that is generally linear (dashed line) for the entire range of beam current and beam speed used for the first build (Fig. 4). Because the beam voltage is constant during the build (60 $\mathrm{kV}$ ), the ratio $I / V$ is proportional to the energy per unit length of travel delivered by the electron beam to the build (where $1 \mathrm{~mA} \cdot \mathrm{sec} / \mathrm{mm}=60 \mathrm{~J} / \mathrm{mm}$ ). The behavior observed in Fig. 4 suggests, therefore, that the mass of powder melted and added to the mesh is roughly proportional to the energy delivered by the electron beam to the build.

Although the simplicity of a linear relationship is especially appealing and convenient for making adjustments to process parameters, extrapolating the dashed straight line in Fig. 4 to zero beam energy has a disturbing implication; namely, a mesh with a mass of $\sim 5 \mathrm{~g}$ will result with the beam off. Clearly this linear model fit to the data has some unphysical consequences.

To understand better the relationship between the mesh mass and the energy delivered by the electron beam, a nonlinear model can be helpful. Mesh structures based on a single contour and built by EBM can be viewed as a stack of melt volumes or voxels-one melt voxel for each strut for each layer of the structure. Ideally, the melt voxel is a right cylindrical solid with a diameter, $d_{v}$, and a thickness, $t_{v}$ , where $t_{v}$ is equal to the layer thickness of the build -in this case $0.05 \mathrm{~mm}$ (see Fig. $5 \mathrm{a}$ ). The volume of the cylindrical melt voxel, $v_{v}$, is, therefore, $\pi t_{v} d_{v}{ }^{2} / 4$, and, if the material density is, $\boldsymbol{\rho}_{0}$, its mass is $\pi \boldsymbol{\rho}_{0} t_{v}$ $d_{v}^{2} / 4$.

To simplify analysis, the energy delivered by the electron beam to the build may be assumed to be bounded by a hemispherical surface. There will be a radial thermal gradient within the hemisphere where temperatures are highest at the center directly beneath the beam. This is schematically represented in Fig. 6 as a spectral gradient where the melting temperature is represented as the transition from yellow-to-green. Because of the large latent heat of fusion, much of the energy delivered by the electron beam will be concentrated within the concentric yellow-to-green hemisphere associated with melting. The total melt volume, $v_{t}$, within this melt hemisphere, consisting of both remelted material and newly melted powder (see Fig. 6 caption), is assumed to be approximately proportional to the energy delivered by the electron beam. The diameter of the melt hemisphere is equal to the diameter of the melt voxel, $d_{v}$, and its volume, $v_{t}$, is $\pi d_{v}{ }^{3} / 12$.

Based on this model, the melt voxel mass is proportional to $d_{v}{ }^{2}$ and the energy delivered by the electron beam per voxel is proportional to $d_{v}^{3}$ (or similarly $d_{v} \propto \sqrt[3]{\text { energy }}$ ). Therefore, the approximate relationship expected between melt voxel mass and the energy delivered by the electron beam is

$$
\text { mass } \propto(\text { energy })^{2 / 3} \propto(I / V)^{2 / 3} \quad \text { Eqn. } 1
$$

Equation 1 should apply both individually to a single melt voxel and collectively to the entire mesh mass. The solid curve in Fig. 3 is the result of a 2-parameter power law fit using nonlinear regression for an equation of the form $y=a x^{b}$ to all the data where $a$ and $b$ are constants. A value for the exponent, $b$, of 
0.682 was determined from the fitting, which agrees well with that expected from Eqn. 1 . Surprisingly, the goodness of fit of the linear and nonlinear models to all the data in this range is the same; $r^{2}=0.98$ in both cases. Although the nonlinear model seems to offer little practical advantage over the linear model for adjusting mesh mass for most beam energies, it excels in accounting for melt behavior at low beam energies.

The third process parameter that was varied in this study and that strongly affects the mesh mass is the focus of the electron beam or focus offset, which is expressed in units of $\mathrm{mA}$ and where a focus offset of $0 \mathrm{~mA}$ corresponds to smallest beam size. A number of mesh cubes were prepared using different focus offsets and beam energies. Results are shown in Fig. 7. For fixed beam energy ( $I / V$ ratio), the mass increases monotonically for increasing focus offset (beam defocus). The defocussed beam distributes energy over more of the powder bed, which leads to more melting of the virgin powder and less re-melting of the underlying strut. At higher focus offsets (highly defocussed beams) and lower beam energies $(I / V)$ the energy density and temperature within the melt voxel are insufficient to melt and fuse the powder, and no mesh is formed. At high relative densities (in this case greater than $\sim 0.27$ ), removing un-sintered powder completely from the mesh after the build and measuring the mesh mass accurately are not possible. Figure 8 shows examples of the range of strut sizes achievable by varying only the focus offset from 0 to $35 \mathrm{~mA}$.

One geometrical consequence of building a mesh structure layer-by-layer with uniform cylindrical melt voxels is the cross-section of a strut taken perpendicular to its long axis (axial crosssection) is not generally circular. Eccentricities of the cross-sections of the struts are intrinsic to this building architecture (see Figs. $5 b$ and $5 c$ ). The strut diameter when viewed along the build direction ( $z$ direction), $d_{\perp}$, is approximately equal to the cylindrical melt voxel diameter, $d_{v}$. However, the strut diameter when viewed perpendicular to the build direction and along the axis of strut tilt, $d_{D}$, is approximately given by,

$$
d_{\mathrm{D}}=d_{v} \sin \theta+t_{v} \cos \theta, \quad \text { Eqn. } 2
$$

where $t_{v} \cos \theta$ must be less than $d_{v} \sin \theta$ in order that the strut be continuous. The aspect ratio of the perpendicular cross-section, $d_{\perp} / d_{\mathrm{D}}$, is

$$
d_{\perp} / d_{D}=\left(\sin \theta+\frac{t_{v}}{d_{v}} \cos \theta\right)^{-1} \quad \text { Eqn. } 3
$$

Eqn. 3 shows that for fixed $\theta$ and $t_{v}$, the aspect ratio of the axial cross-section of the strut increases with increasing melt voxel diameter, $d_{v}$ (see Fig. $5 \mathrm{~d}$ ). This trend is consistent with the mesh eccentricities shown in Fig. 9, namely for higher relative densities (and strut diameters), higher eccentricities are generally observed.

Figure 10 shows two perpendicular views of a strut built with a focus offset of $40 \mathrm{~mA}$, a relative density of $\sim 0.17$, and $\theta=30^{\circ}$. The strut diameter in Fig. $10 \mathrm{a}, d_{\mathrm{D}}$, is $\sim 1.3 \mathrm{~mm}$ and in Fig. $10 \mathrm{~b}, d_{\perp}$, is $\sim 1.7$ $\mathrm{mm}$. For these diameters, Eqn. 3 gives an effective value of $t_{v}$ of $0.52 \mathrm{~mm}-$ a value ten times larger than the machine layer spacing $(0.050 \mathrm{~mm})$. This suggests that the electron beam penetrates and melts 
powder to depths substantially deeper than the machine layer spacing which in turn reduces the aspect ratio of the struts for a given build condition.

Figure 11 shows an optical micrograph of the polished and chemically etched cross-section of a terminal strut of the mesh shown in Figure 10. Clearly evident in the image are a) the roughly hemispherical profile of the terminal melt volume and b) the remnant arcs of the edges of underlying melt volumes spaced apart by roughly the layer thickness $(0.050 \mathrm{~mm})$. The depth of the terminal melt volume in the image is $0.47 \mathrm{~mm}$, which agrees well with the value estimated from the strut eccentricity and Eqn. 3 above $(0.52 \mathrm{~mm})$.

Other notable features of the anisotropic microstructure in Figure 11 include spherical porosity ranging in diameter from approximately 0.01 to $0.2 \mathrm{~mm}$; this is the result of gas originally contained within the rotary atomized spherical powder. Both the upper and lower surfaces of the strut are irregular with the lower surface showing a significant number of partially melted or sintered spherical powder particles. Also, the acicular microstructure, which generally appears to extend radially from the melt volume, shows a concentration that is parallel and above the centerline of the strut.

Both microstructure and eccentricities of the cross-sections of the struts likely contribute to anisotropies in physical properties of the mesh structure. To examine anisotropy and the role of beam parameters in determining mechanical properties, some mesh cubes were tested in compression between two parallel hardened steel plates at a rate of $76 \mathrm{~mm} / \mathrm{min}$ using a standard MTS Systems loading apparatus. Figure 12 shows the results for two nominally identical mesh cubes with different orientations of loading. The behavior is similar for both cubes and is typical for mesh and foam structures [5]. For low loads, the stress-strain relationship is linear and struts undergo reversible elastic bending. For moderate loads, a stress plateau is observed where the plateau stress, $\sigma_{p l}$, is nearly constant and the mesh structure irreversibly collapses while struts buckle and deform. For the highest loads, the struts of the collapsed mesh structure impinge upon each other and the mechanical behavior rapidly approaches that of an array of stacked struts.

Figure 12 shows a difference in mechanical behavior of the mesh with load orientation. For loading parallel to the build direction, both the plateau yield stress $\left(\sigma_{p l}\right)$ and the linear elastic modulus ( $E^{*}$ or the nearly constant slope of the stress-strain curve for low strains) are higher than for loading perpendicular to the build direction. These differences may be the result of the directionally oriented microstructure that is observed in Fig. 11 and is typically characteristic of the AM-EBM process [6]. In order to determine whether these differences for mesh are statistically significant, several pairs of identical mesh cubes were prepared using a range of build parameters and tested in two load orientations.

For load along the build direction, Fig. 13 shows some typical results for mesh cubes prepared using zero focus offset and different beam current and beam speed. By varying only the beam current and beam speed, large variations in both $\sigma_{p l}$ and $E^{*}$ are clearly achievable.

Figure 14 shows a compilation of the relative elastic moduli $\left(E^{*} / E_{0}\right)$ and the relative plateau yield stresses $\left(\sigma_{p l} / \sigma_{0}\right)$ versus the relative density $\left(\boldsymbol{\rho}^{*} / \boldsymbol{\rho}_{0}\right)$ for sixteen mesh cubes tested in compression 
perpendicular and parallel to the build direction. Here $E_{0}$ and $\sigma_{0}$ are the elastic or Young's modulus ( $210 \mathrm{GPa})$ and yield strength ( $1.35 \mathrm{GPa}$ ) for bulk Inconel 625 at room temperature. The data show that the values of both the relative elastic modulus and the relative yield stress tend to be higher for loads applied parallel to the build direction than perpendicular to the build direction. Interestingly for lower relative densities (which correspond to lower melt voxel diameters, lower $d_{v} / t_{v}$, and lower strut aspect ratios), the differences in values for parallel and perpendicular loads are greater. This higher anisotropy of mechanical behavior is contrary to that expected from Eqn. 3 for struts with a lower aspect ratio of cross-section. The cause of this behavior appears not to be simply strut eccentricity but is likely related to microstructural differences such as a) the degree of strut melting and the distribution of strut porosity observed in Fig. 9 or b) the volume fraction of the acicular phase (Fig. 11). Further microstructural analysis is required to understand the cause of trends in mechanical anisotropy with relative density (i.e., strut dimensions) shown in Figure 14.

Straight lines have been fit to the data in Fig. 14 and have average slopes of 1.9 for both the elastic modulus and yield stress. These values are within a reasonable range of those expected for open-cell and foam structures (1.5 for yield stress and 2.0 for elastic modulus) $[5,7]$ and are consistent with those reported by others for Inconel 625 as well as other alloys and pure metals $[5,6,8]$.

Figure 14 shows that for an Inconel 625 mesh cube both its modulus and yield strength can be systematically varied by greater than a factor of ten by adjusting only electron beam parameters. This suggests that with proper control software, a single mesh structure can be fabricated layer-by-layer with AM-EBM such that its physical properties can be specified on a scale approaching the machine layer spacing. This capability, to fabricate fine-scale functionally graded structures, is of immediate interest within the additive manufacturing community $[1,4,9]$.

\section{Summary}

Some relationships between electron beam parameters (beam current, beam speed, and beam focus) and physical properties (mass, diameter, elastic modulus, and yield strength) have been investigated for Inconel 625 mesh cubes fabricated using AM-EBM. The elastic modulus and yield strength of the mesh cubes have been systematically varied by approximately a factor of ten by changing the electron beam parameters. Simple models have been used to understand these relationships. Structural anisotropies of the mesh associated with the layered build architecture have been observed and may contribute, along with observed microstructural anisotropies, to the anisotropic mechanical properties of the mesh. Knowledge of this kind is likely applicable to other metal and alloy AM-EBM systems and is essential to rapidly realize the full potential of this burgeoning technology. Future work in this area might include a) quantifying porosity and microstructure within individual struts, b) testing tensile properties, c) exploring effects of annealing on mechanical properties, and d) comparing mechanical properties to those based on calculations or simulations. 


\section{Acknowledgements}

Research sponsored by the U.S. Department of Energy, Office of Energy Efficiency and Renewable Energy, Advanced Manufacturing Office, under contract DE-AC05-00OR22725 with UT-Battelle, LLC.

\section{References}

[1] Parthasarathy, J., Starly, B., Raman, S., and Christensen, A.: J. Mech. Behav. Biomater., 2010, 3, 249.

[2] Harrysson, O.L.A., Cansizoglu, O., Marcellin-Little, D.J., Cormier, D.R., and West II, H.A.: Mater Sci Engr. C, 2008, 28, 366.

[3] Campoli, G., Borleffs, M.S., Amin Yavari, S., Wauthle, r., Weinans, H., and Zadpoor, A.A.: Mater. Des., 2013, 49, 957.

[4] Murr, L.E., Gaytan, S.M., Medina, F., Lopez, H., Martinez, E., Machado, B.I., Hernandez, D.H., Martinez, L., Lopez, M.I., Wicker, R.B., and Bracke, J.: Phil. Trans. R. Soc. A, 2010, 368, 1999.

[5] Gibson, L.J.: Ann. Rev. Mater. Sci., 2000, 30, 191.

[6] Murr, L.E., Gaytan, S.M., Ramirez, D.A., Martinez, E., Hernandez, J., Amato, K.N., Shindo, P.W., Medina, F.R., and Wicker, R.B.: J. Mater. Sci. Technol., 2012, 28 (1), 1.

[7] Gibson, L.J. and Ashby, M.F.: Cellular Solids: Structure and Properties, Cambridge Univ. Press, New York, 1997.

[8] Heinl, P., Müller, L., Körner, C., Singer, R.F., and Müller, F.A.: Acta Biomater., 2008, 4, 1536.

[9] Heinl, P., Körner, C., and Singer, R.F.: Adv. Eng. Mater., 2008, 10, 882. 
a
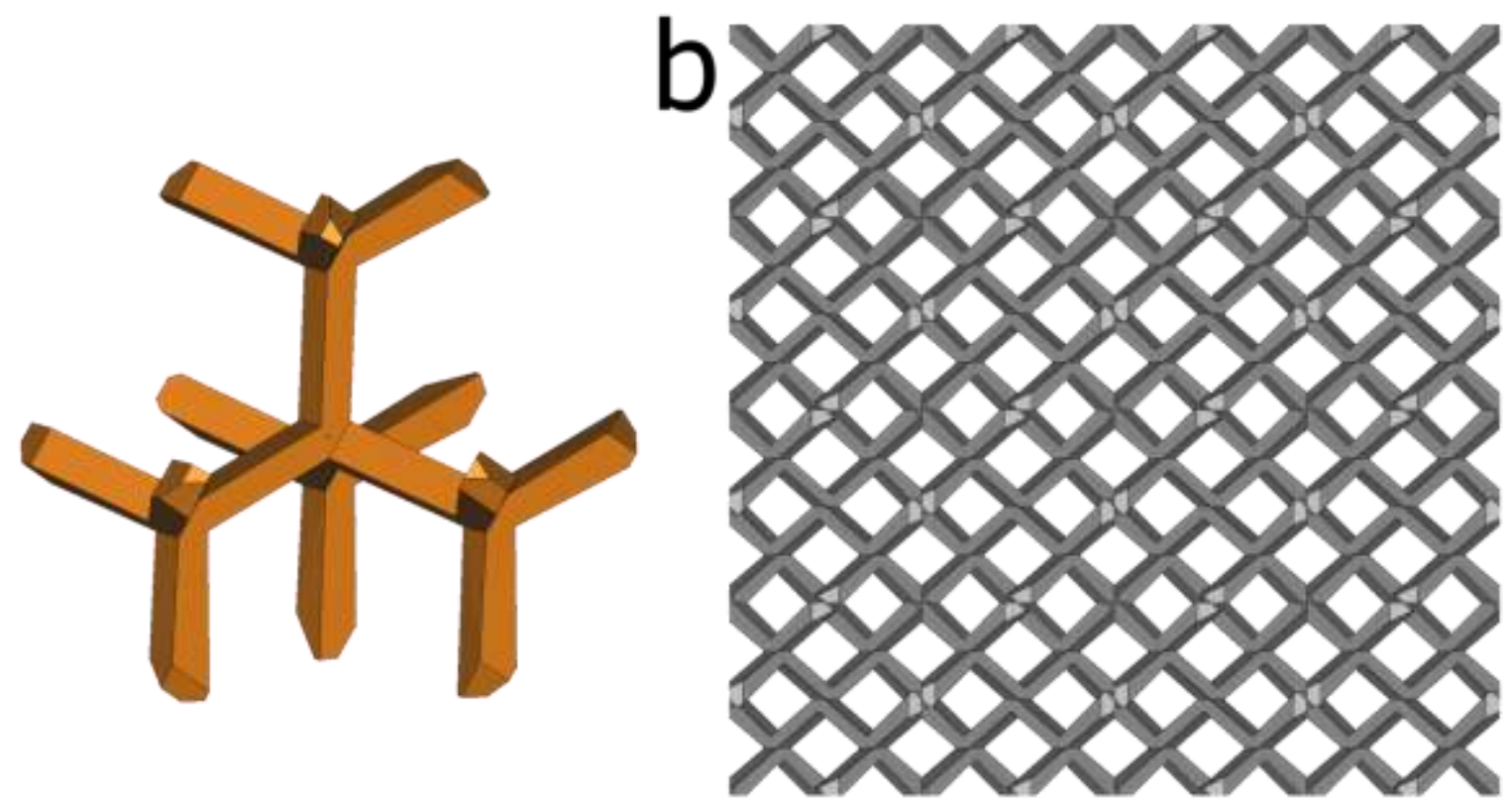

Figure 1: a) Model of the diamond mesh unit cell, and b) mesh cube model viewed perpendicular to the cube face.

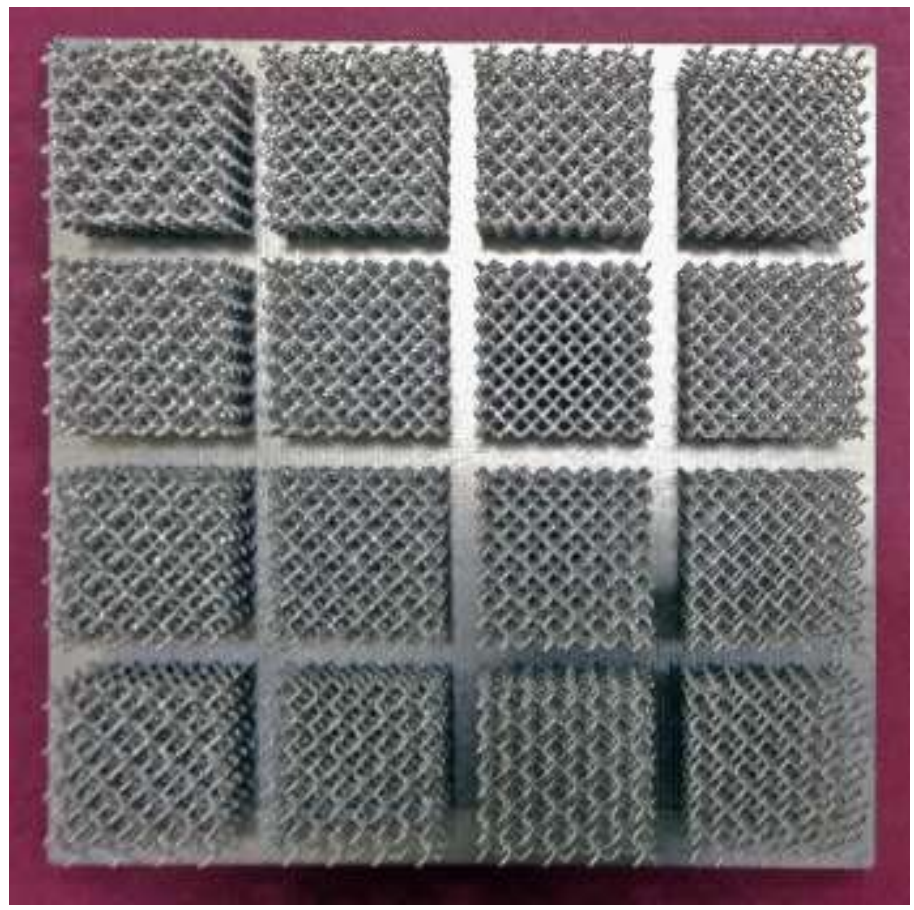

Figure 2: Photograph of the top view of the first build of sixteen mesh cubes. 


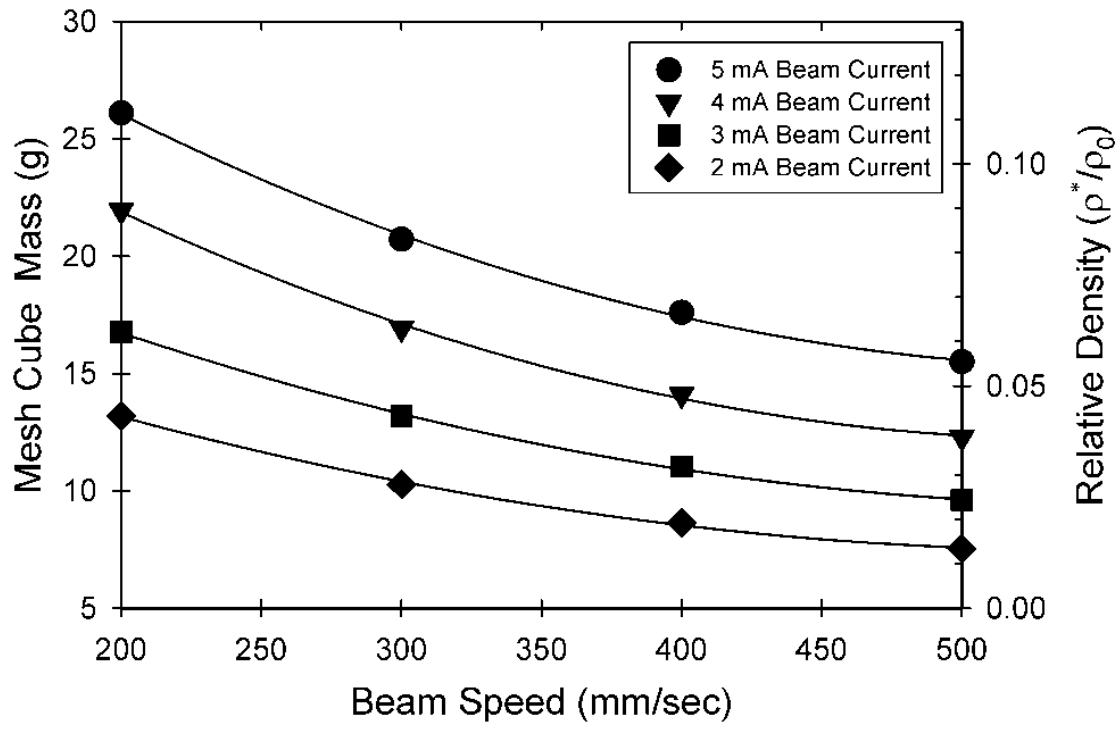

Figure 3: Mesh cube mass versus beam speed for the first build. Lines are to guide the eye.

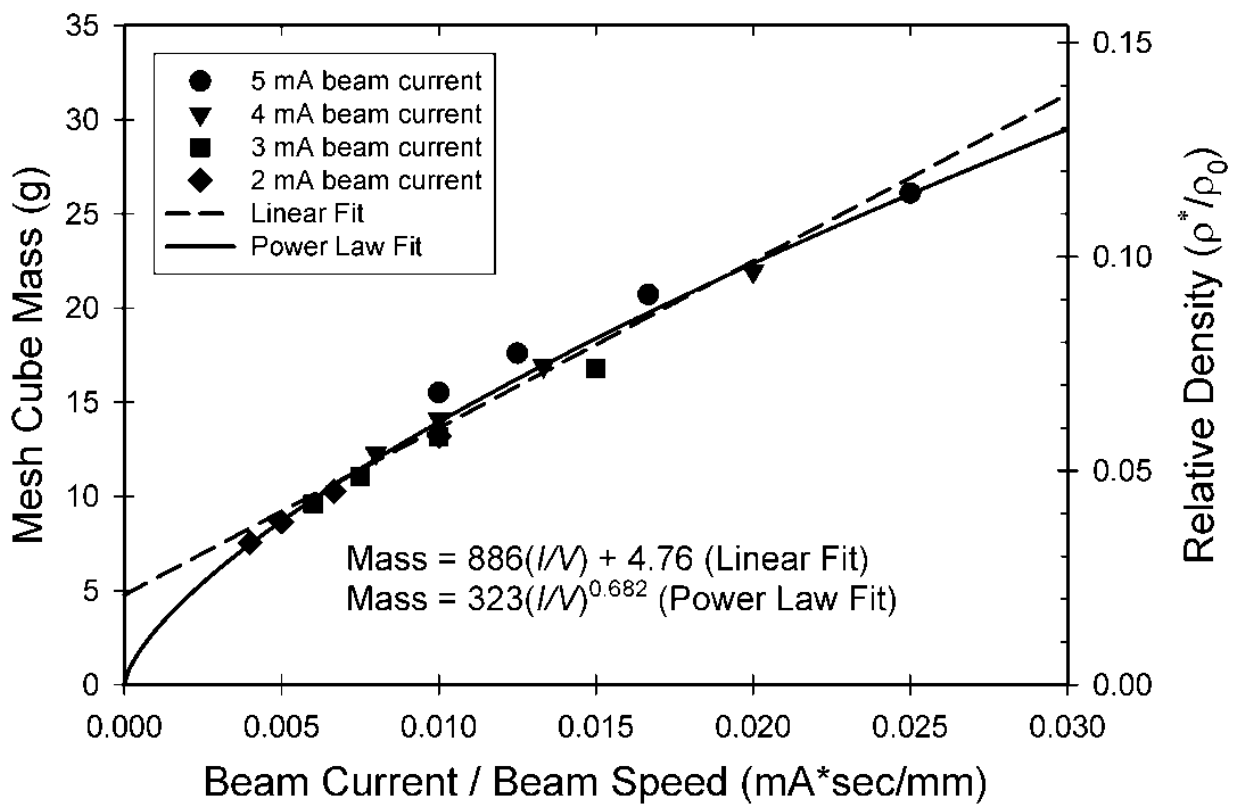

Figure 4: Mesh cube mass versus current to speed ratio $(I / V)$ for $0 \mathrm{~mA}$ focus offset current. The dashed curve is a straight line fit to all the data and extended to the axes, and the solid curve is a power law fit to all the data (see text). 


\section{a}
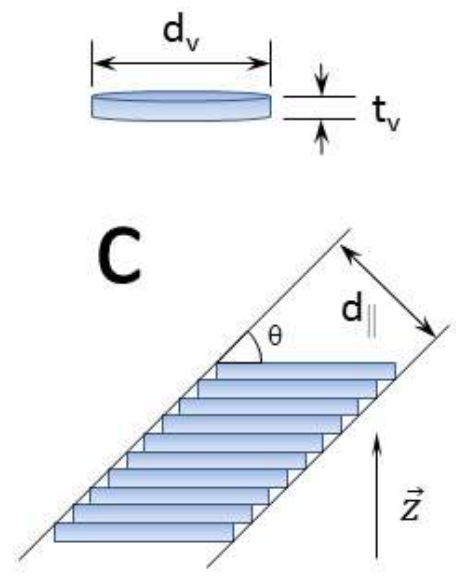
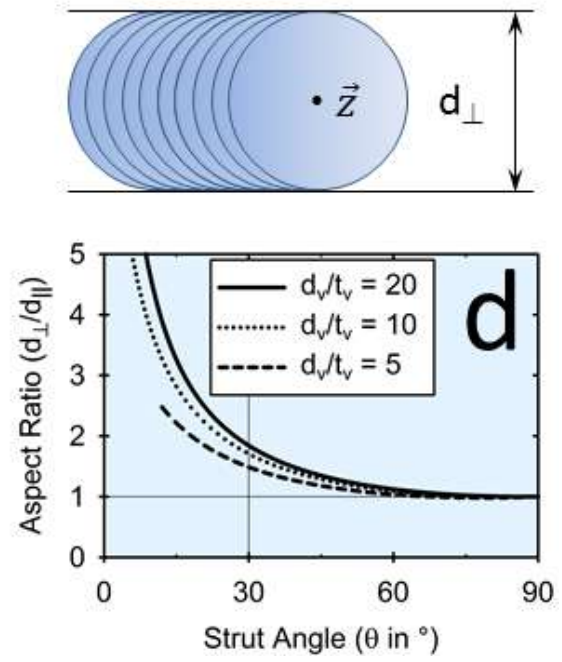

Figure 5: Some mesh geometries for a cylindrical melt voxel model; a.) ideal melt voxel, b.) top view of a strut stack, c.) side view of a strut stack, and d.) aspect ratio $\left(d_{\perp} / d_{D}\right)$ of the perpendicular cross-section of the strut for various $d_{v} / t_{v}(s e e$ text). The vector $z$ in $c$.) is parallel to the AM-EBM build direction.

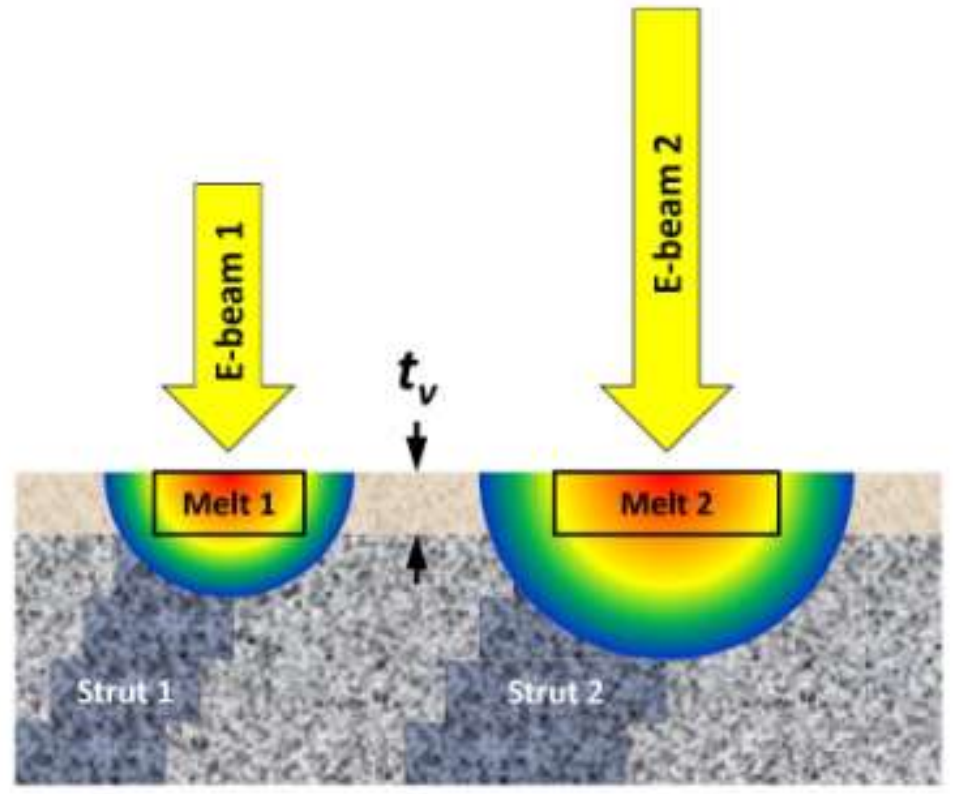

Figure 6: A simple melt voxel model for AM-EBM mesh where e-beam 2 delivers more energy than e-beam 1. The transition from yellow-to-green is considered the melting temperature. The rectangles labelled Melt 1 and Melt 2 represent the volumes of newly melted powder. 


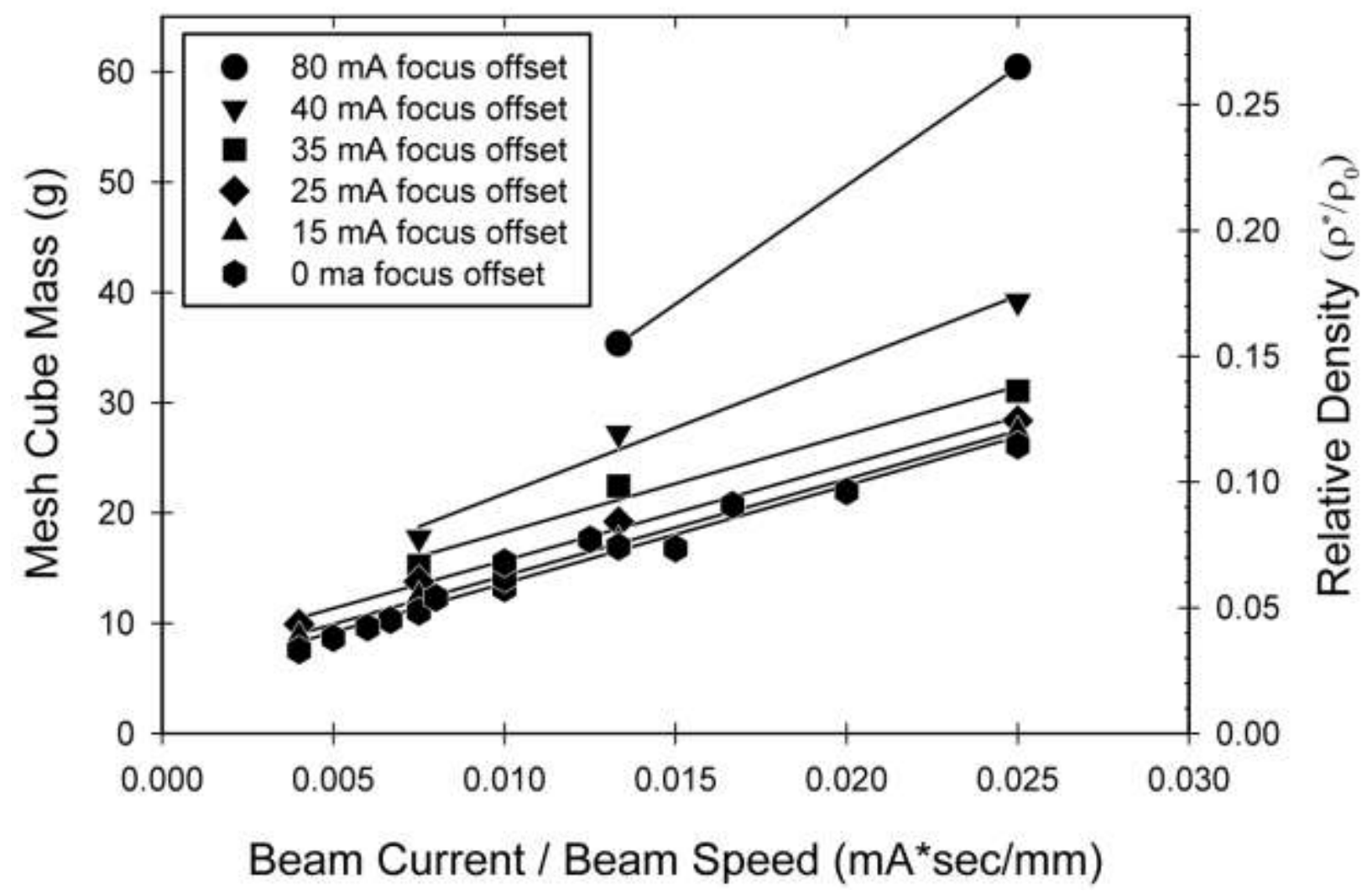

Figure 7: Mesh cube mass versus $I / V$ for various focus offset currents. Straight lines are fit to data for each focus offset current to guide the eye.

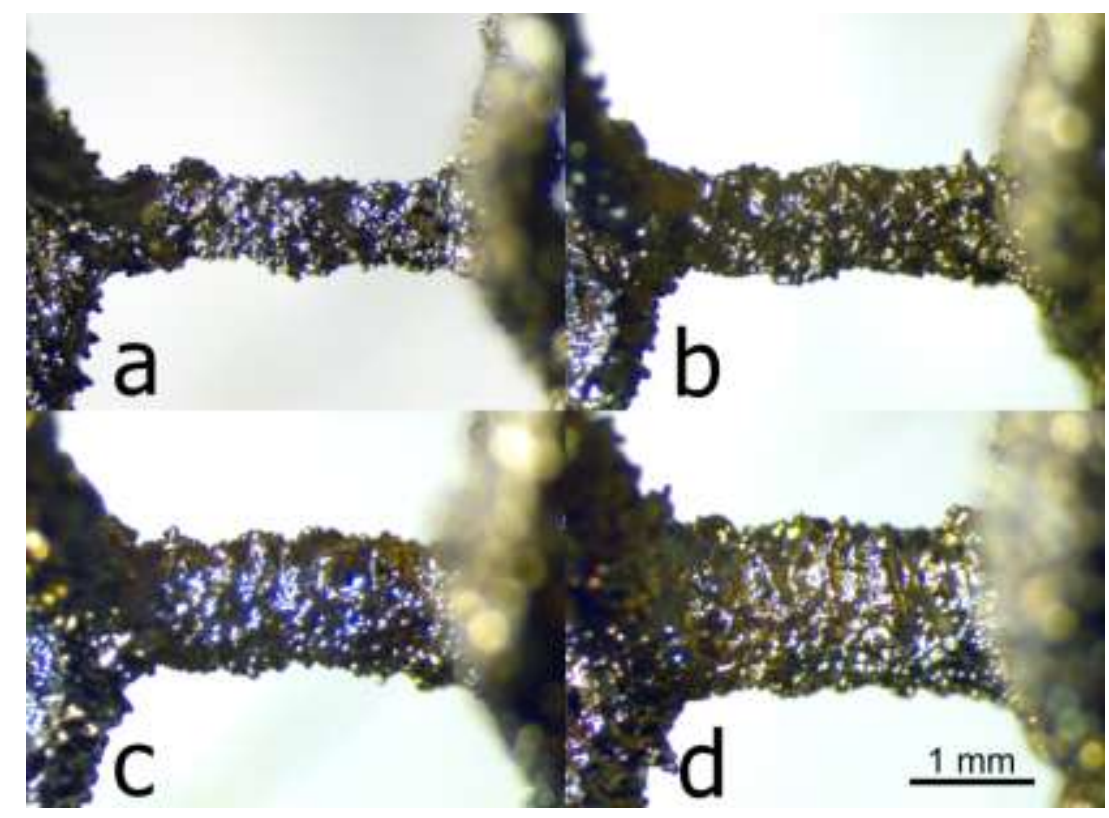

Figure 8: Four struts viewed parallel to the build direction for different focus offset currents; a) $0 \mathrm{~mA}, \mathrm{~b}) 15 \mathrm{~mA}, \mathrm{c}) 25 \mathrm{~mA}$, and d) $35 \mathrm{~mA}$. The $I / V$ is equal to $\sim 0.025 \mathrm{~mA} * \mathrm{sec} / \mathrm{mm}$ for all struts. 


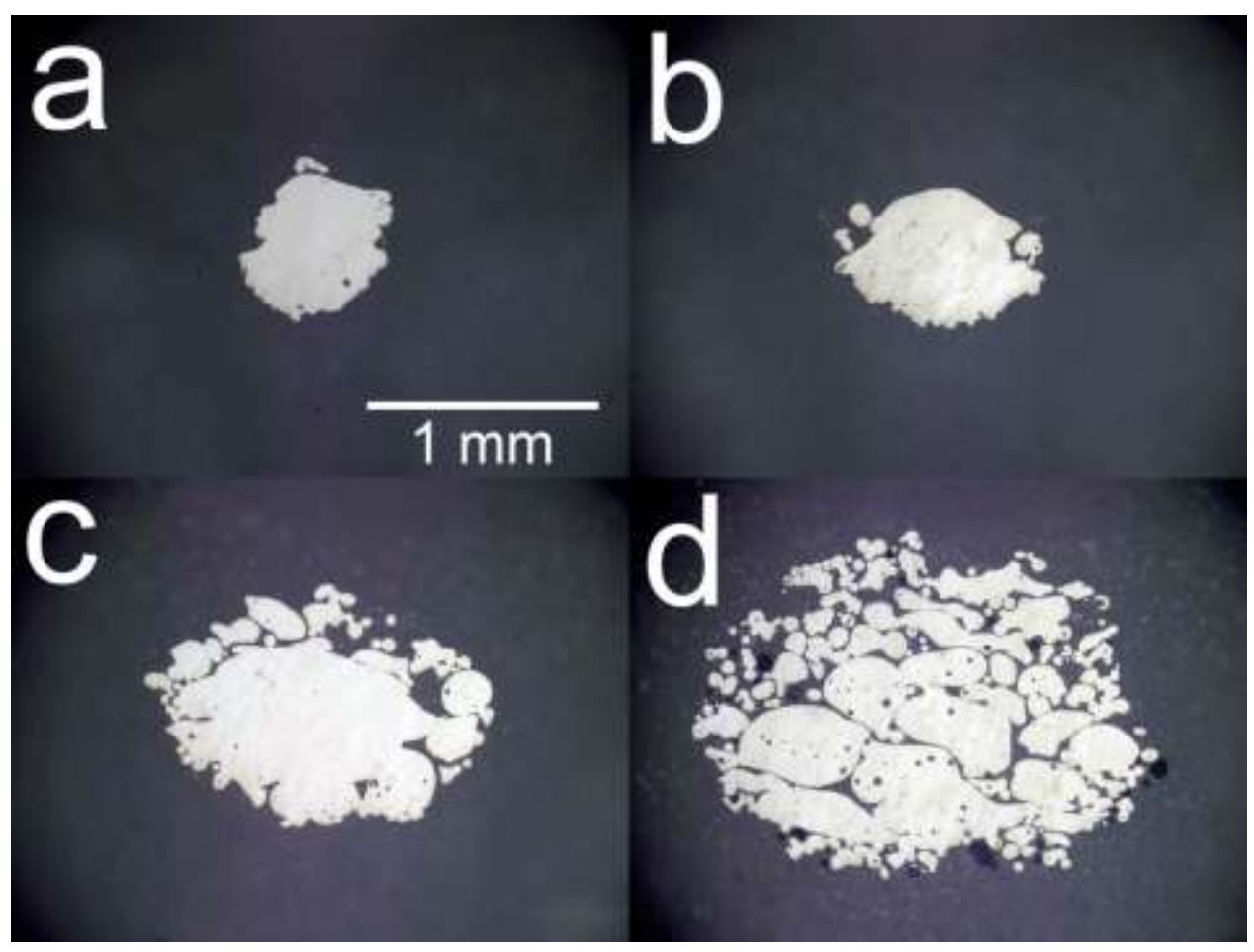

Figure 9: Four axial views of struts of meshes of different relative densities; a) $\rho^{*} / \rho_{0}=0.054$, b) $\rho^{*} / \rho_{0}=0.079$, c) $\rho^{*} / \rho_{0}=0.16$, and d) $\rho^{*} / \rho_{0}=0.26$. Higher relative density meshes generally show greater aspect ratios and less complete melting.

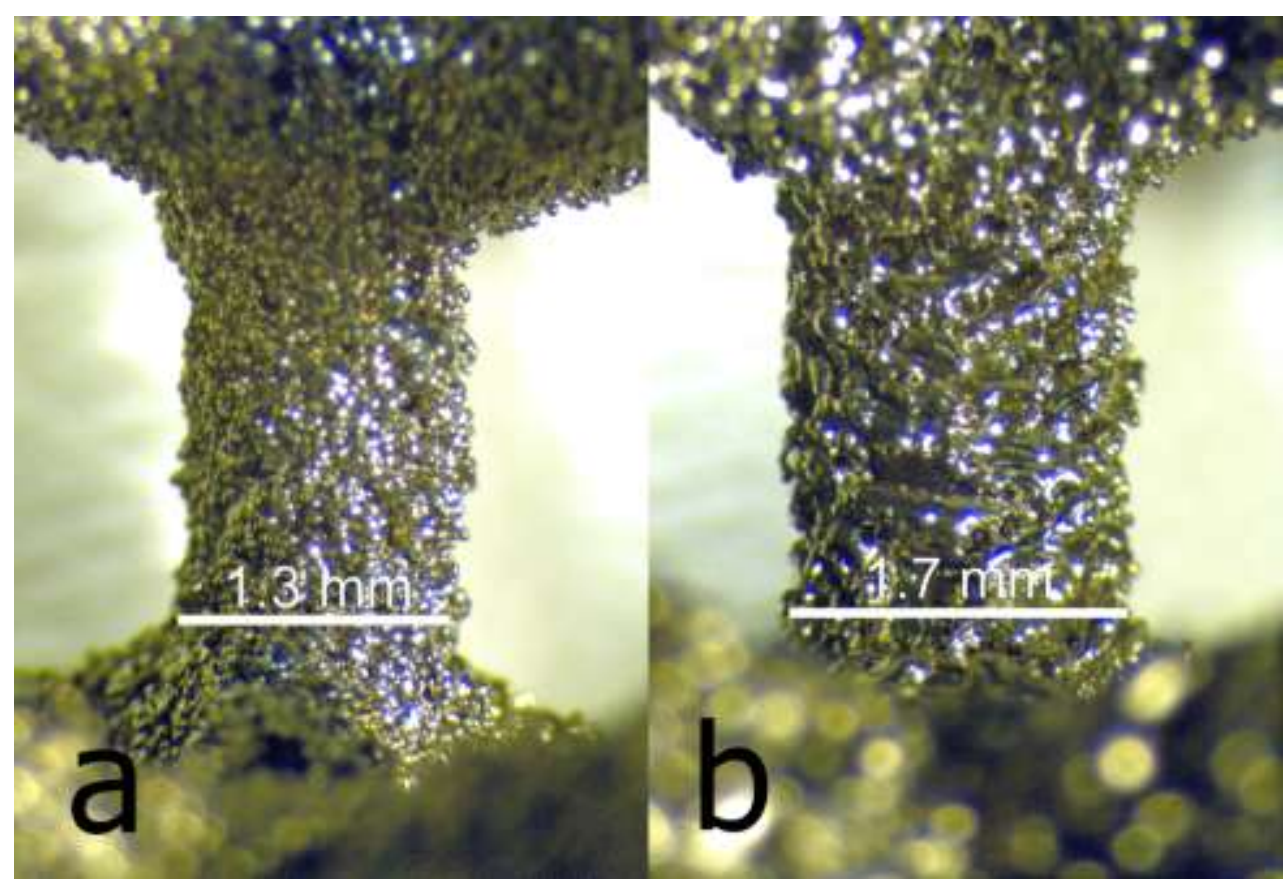

Figure 10: Two views of the same strut; a) perpendicular to the build direction and along the axis of strut tilt and b) parallel to the build direction. Here the focus offset is $40 \mathrm{~mA}$, and $I / V$ is $\sim 0.025 \mathrm{~mA} * \mathrm{sec} / \mathrm{mm}$. 


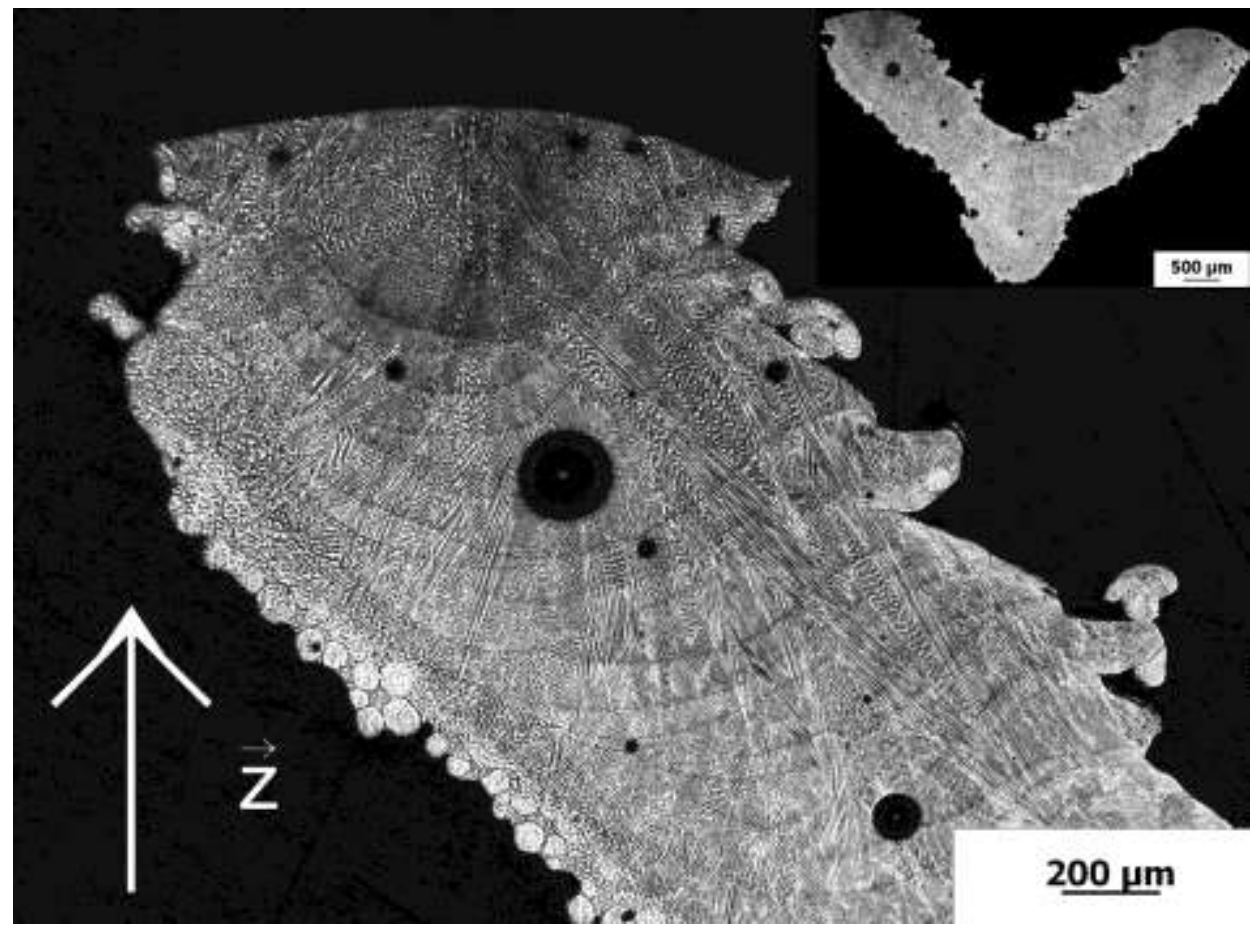

Figure 11: Optical micrograph of the polished and chemically etched cross-section of a terminal strut of the mesh shown in Figure 10. The inset (upper right) shows the entire strut and its companion.

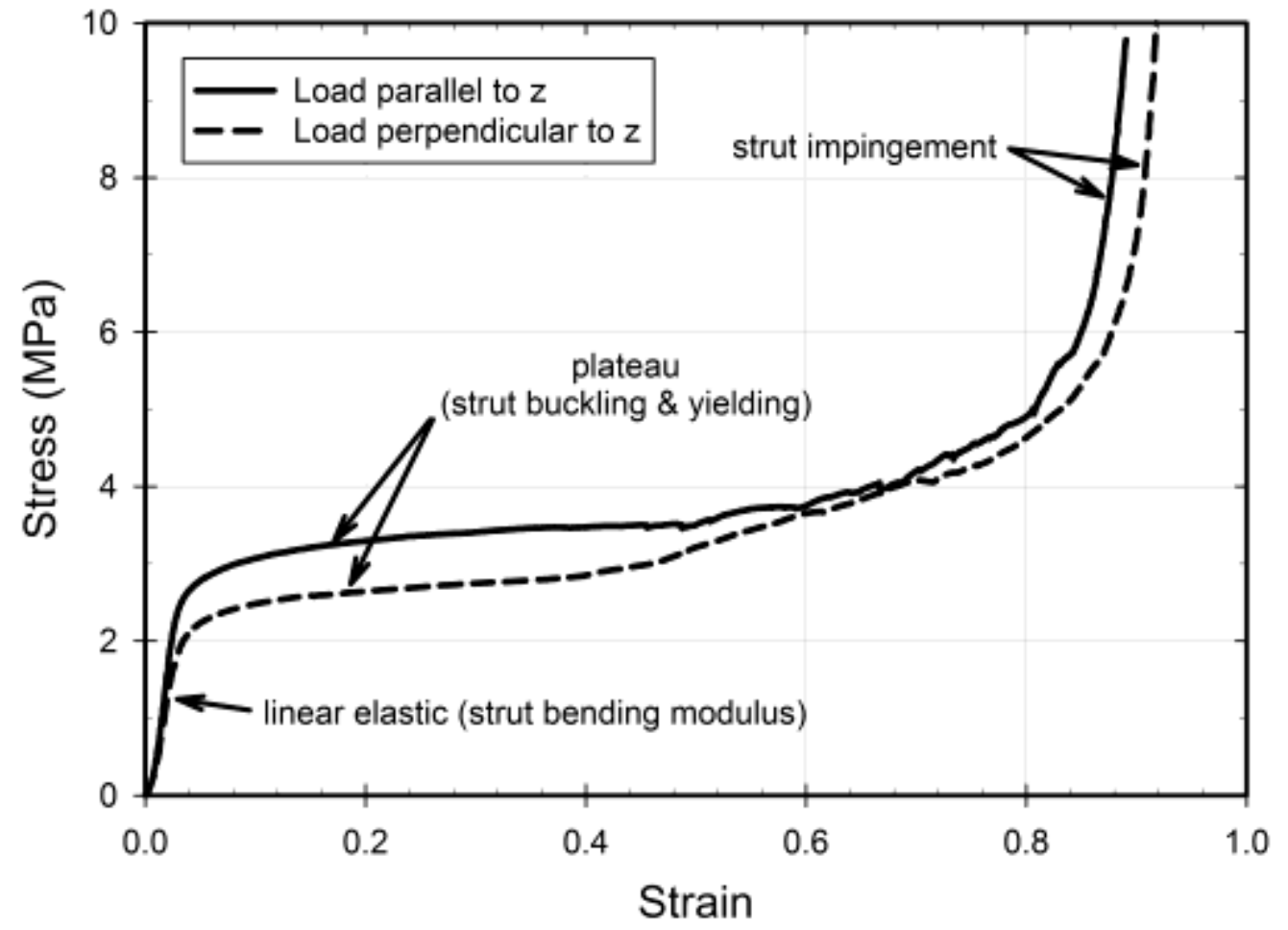

Figure 12: Engineering stress versus strain for two mesh cubes with diffent orientations of compressive loading. Both cubes were fabricated using identical build conditions and have a relative density of $\sim 0.053$. 


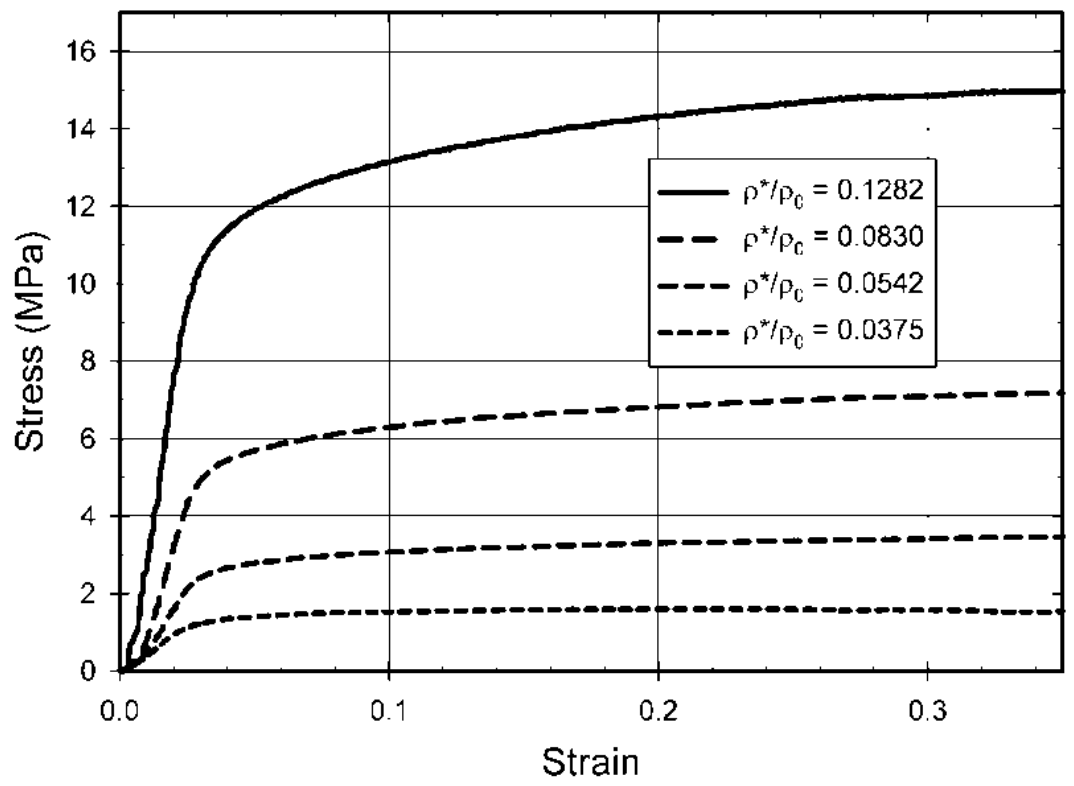

Figure 13: Engineering compressive stress versus strain for mesh cubes with a range of mesh densities. For these data, the load is applied parallel to the build axis (z). 


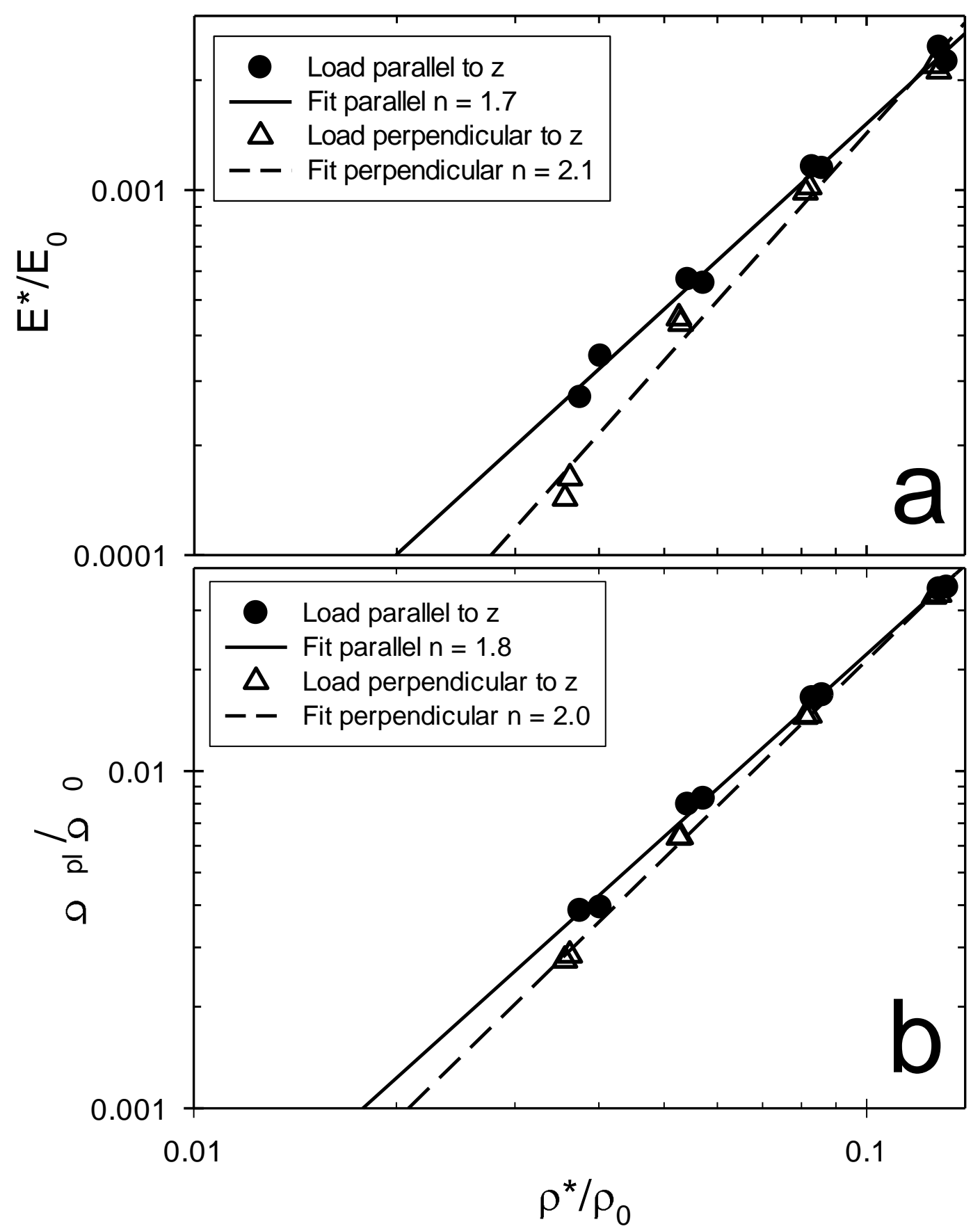

Figure 14: Relative elastic modulus $\left(E^{*} / E_{0}\right)$ and relative plateau yield stress $\left(\sigma_{p l} / \sigma_{0}\right)$ versus relative density $\left(\rho^{*} / \rho_{0}\right)$ for cubes loaded in compression both parallel and perpendicular to the build axis (z). The lines are fit to the data (see text). 